\title{
SEGNALAZIONI FAUNISTICHE ITALIANE
}

\section{2 - Cerambyx cerdo Linnaeus, 1758 (Coleoptera, Cerambycidae)}

Rastelli S., Abruzzese E., Rastelli M., 2001 - Cerambycidae d'Italia. Atlante fotografico dei Coleotteri Cerambicidi italiani.Museo Civico di Storia Naturale di Carmagnola, Centro Studi Ecologici Appenninici. CD ROM.

Prima segnalazione per l'isola di Montecristo di specie turano-europeo-mediterranea a distribuzione assai frammentata in Italia.

RePerti. Toscana: isola di Montecristo, $400 \mathrm{~m}$ a N di Punta dei Lecci (Portoferraio, Livorno), 360 s.l.m., UTM WGS84 32T60784687, 17.IX.2019, 1 ô e 1 elitra S. Piazzini leg. e det. (coll. S. Piazzini, Casole d'Elsa, SI).

OSSERVAZIONI. Specie a corotipo turano-europeo-mediterraneo nota in Italia in tutta la penisola e nelle isole maggiori ma con popolazioni assai frammentate (Sama, 2007; Stoch \& Genovesi, 2016). In Toscana è distribuita in tutte le province ad eccezione di quella di Massa-Carrara (Sforzi, 2001; Terzani et al., 2011). Si tratta di una specie xilofaga legata esclusivamente a querceti (cerrete, sugherete, leccete, ecc.) vetusti o con presenza di piante morte o deperienti, che si sviluppa su tronchi o grossi rami di piante senescenti. In tutto l'areale è in progressiva rarefazione per la scomparsa dell'habitat causata della semplificazione della struttura dei boschi dovuta al taglio e alla rimozione delle piante secche o deperienti (Albert et al., 2012) e per le persecuzioni dirette, perché considerato dannoso per i querceti (Campanaro et al., 2011). Per queste ragioni è considerato "Vulnerable" a livello globale (IUCN, 2019) ed è inserito negli allegati II e IV della Direttiva 92/43/CEE e nell'allegato A della L.R. Toscana 56/2000. Cerambyx cerdo è da ritenersi a rischio nell'isola di Montecristo, nonostante il territorio sia rigorosamente protetto da una Riserva Integrale, visto che la popolazione di leccio (Quercus ilex) alla quale risulta infeudata ammonta a soli 208 esemplari (Crudele et al., 2005), in buona parte ultracentenari e senescenti, e che, a causa del sovrapascolo operato dai molti esemplari di capra selvatica (Capra hircus), la rinnovazione è sporadica e limitata ad alcune recinzioni realizzate nell'area di Collo dei Lecci nell'ambito del progetto LIFE NAT/IT/000353 “Montecristo 2010”.

\section{BIBLIOGRAFIA}

Albert J., Platek M., Cizek L., 2012 - Vertical stratification and microhabitat selection by the Great Capricorn Beetle (Cerambyx cerdo) (Coleoptera: Cerambycidae) in open-grown, veteran oaks. European Journal of Entomology, 109(4): 553-559.

Campanaro A., Bardiani M., Spada L., Carnevali L., Montalto F., Antonini G., Mason F., Audisio P., 2011 - Linee Guida per il monitoraggio e la conservazione dell'entomofauna saproxilica / Guidelines for monitoring and conservation of saproxylic insects. Quaderni Conservazione Habitat, 6, Verona, pp. 44. + CD-ROM.

Crudele G., Landi M., Zoccola A., 2005 - La popolazione di Quercus ilex L. nella Riserva Naturale Biogenetica Isola di Montecristo: osservazioni, considerazioni e interventi di conservazione. (Magnoliopsida Fagales Fagaceae). Quaderno di Studi e Notizie di Storia Naturale della Romagna, 21: 59-89.

IUCN, 2019 - The IUCN Red List of Threatened Species. Version 2019-2. https://www.iucnredlist.org.

SAma G., 2007. Coleoptera Cerambycidae. In: Ruffo S., Stoch F. (eds). Checklist and distribution of the italian fauna. Ministero dell'Ambiente. CD ROM.

Sforzi A., 2001 - Cerambycidae (pp. 229-242). In: Sforzi A., BARTOlozzi L. (a cura di). Libro Rosso degli Insetti della Toscana. Agenzia Regionale per lo Sviluppo e l'Innovazione nel settore Agricolo-forestale, Firenze, pp. 375.

Stoch F., Genovesi P., 2016 - Manuali per il monitoraggio di specie e habitat di interesse comunitario (Direttiva 92/43/CEE) in Italia: specie animali. ISPRA, Serie Manuali e linee guida, 141/2016.

Terzani F., Cianferoni F., Fabiano F., Mazza G., Monte C., Zinetti F., Ballerini C., 2011 - Coleotterofauna della Provincia di Prato (Toscana): elenco preliminare (Coleoptera). Onychium, 8: 22-76.

Sandro PIAZZINI, Leonardo FAVILLI

Dipartimento di Scienze Fisiche, della Terra e dell'Ambiente, via Mattioli 4, I-53100 Siena (SI), Italia.

E-mail: piazzini5@unisi.it

Giovanni QUILGHINI

Reparto Carabinieri per la Biodiversità di Follonica (GR), via Bicocchi 2, I-58022 Follonica (GR), Italia. 


\section{3 - Apterola kuenckeli kuenckeli Mulsant \& Rey, 1866 (Heteroptera Lygaeidae)}

PERICART J., 1999 - Hémiptères Lygaeidae euro-méditerranéens vol.1 - Faune de France et régiones limitrophes 84A: 139 -141. Fédération Fracaise de Sociétés de Sciences Naturelles, Paris.

Prima segnalazione per la Puglia e nuova località per l'Abruzzo di specie mediterranea a diffusione localizzata, nota per alcune regioni meridionali e centrali, nonché per varie aree insulari italiane.

REPERTI. Puglia: prov. Foggia, Chieuti, località Marina di Chieuti, m 3 s.l.m., su muro presso massicciata ferroviaria, 28.VI.2017, N. Olivieri leg., 2 ふึત 2 우 (det. et coll. Olivieri).

Abruzzo: prov. Chieti, Ortona, m 4 s.l.m., su muro presso massicciata ferroviaria, 13.VIII.2016, N. Olivieri leg., 2 ๙ิํㅗ 3 우우; (det. et coll. Olivieri).

OSSERVAZIONI. Specie a geonemia mediterranea, suddivisa in quattro sottospecie di cui A. kuenckeli kuenckeli è diffusa in Spagna meridionale e orientale, Francia meridionale, Corsica, Italia meridionale, Sicilia, Sardegna, Malta, Grecia continentale, Repubblica di Macedonia, Bulgaria, Marocco, Algeria settentrionale, Tunisia e Libia nordorientale, A. kuenckeli rubicunda (Stål, 1872) è presente a Cipro, in Turchia meridionale e centrale, Siria, Libano, Israele, Giordania e Iran, A. kuenckeli obscura Deckert 1995 è nota dell'Andalusia e del Marocco occidentale e A. kuenckeli focarilei Tamanini 1964 è esclusiva di Pantelleria (Pericart, 1999). La specie è stata rinvenuta in Italia in Abruzzo (Luigioni, 1929; Tamanini, 1964, 1973), Lazio (Natura Mediterraneo, 2009) Campania (Pericart, 1999), Basilicata (Tamanini, 1981), Calabria (Tamanini, 1964, 1981) Sicilia (Walker, 1872; Costa, 1887; Oshanin, 1909; Stichel, 1938; Mancini, 1952, 1960; Wagner, 1958, Tamanini, 1964; Slater, 1964; Servadei, 1967), Sardegna (Costa, 1883; Ferrari, 1888; Singer \& Mancini, 1938; Servadei, 1952; Stichel, 1959; Mancini 1960; Tamanini, 1964; Slater, 1964), Isole Eolie (Tamanini, 1981), Lampedusa (Mancini, 1960) e Pantelleria (Tamanini, 1964). Apterola kuenckeli kuenckeli è una specie attera, legata alle zone particolarmente xerotermiche della regione mediterranea, dove vive sul suolo o sotto le pietre e localmente può essere numerosa. Nella località di rinvenimento nella Puglia settentrionale, a Chieuti, la specie compare con un elevato numero di esemplari su un basso muro in cemento prossimo alla massicciata ferroviaria, tale muro è fiancheggiato da un incolto arido occupato da vegetazione erbacea soggetta a disturbo antropico, sviluppatasi su pietrisco calcareo di riporto. Gli individui in orario pomeridiano tendono ad aggregarsi intorno a possibili fonti alimentari, rappresentate da residui vegetali e deiezioni di uccelli. In Abruzzo, ad Ortona, la specie è stata rinvenuta in alcuni individui, ugualmente su un basso muro in cemento, situato presso la massicciata ferroviaria, in una zona caratterizzata da prati aridi soggetti a disturbo antropico, posti a circa $100 \mathrm{~m}$ di distanza dal mare. In Abruzzo la specie era già stata segnalata da Luigioni (1929) a Pescasseroli (L'Aquila), presso il Rifugio della Difesa, a circa 1270 m s.l.m. di quota, con un esemplare trovato sotto una pietra; su tale ritrovamento si basano anche le citazioni di Tamanini $(1964,1973)$ per la regione. La nuova località di rinvenimento della specie in Abruzzo, che si trova a circa $107 \mathrm{~km}$ di distanza in linea d'aria dal luogo della prima osservazione, in una zona situata presso il mare Adriatico e soggetta a clima mediterraneo, amplia significativamente l'area di presenza della specie nella regione. Il ritrovamento di Apterola kuenckeli kuenckeli nelle due località costiere situate nella Puglia settentrionale e in Abruzzo, a circa $94 \mathrm{~km}$ di distanza tra loro, testimonia la diffusione della specie anche lungo il versante adriatico della penisola italiana.

\section{BIBLIOGRAFIA}

Costa A., 1883 - Notizie ed osservazioni sulla geofauna sarda. Memoria Seconda. Risultamento di ricerche fatte in Sardegna nella primavera del 1882, Atti della Reale Accademia delle Scienze Fisiche e Matematiche di Napoli ser. 2, 1(2): 1-109.

Costa A., 1887 - Lettera al chiarissimo signor Enrico Ragusa entomologo a Palermo. Il Naturalista Siciliano, 6: $228-229$.

FERrARI, P.M. 1888 - Elenco dei Rincoti sardi che si trovano nella collezione del Museo Civico di Genova. Annali del Museo Civico di Storia Naturale di Genova ser. 2, 6: 545-560.

Luigioni P., 1929 - Primo contributo alla conoscenza della fauna entomologica del Parco Nazionale d'Abruzzo - Emitteri. Acta Pontificia Accademia delle Scienze, Nuovi Lincei, 82: 201-220.

MANCINI C. 1952 - Miscellanea emitterologica italiana. I. Bollettino della Società Entomologica Italiana, 82: 56-64.

MANCINI C. 1960 - Hemiptera in Zavattari et al. (eds) Biogeografia delle Isole Pelagie. Rendiconti dell'Accademia Nazionale dei XL, 11: 335 - 341. 
NATURA MEDiterRANEO, 2009 https://www.naturamediterraneo.com/forum/topic.asp?whichpage=0,6\&TOPIC_ID=69908\&

OshanIN B., 1909 - Verzeichnis der Paläarktischen Hemipteren mit besonderer Berücksichtigung ihrer Verteilung im Russischen Reiche. Bd. I Heteroptera. Buchdruckerei der Kaiserlichen Akademie der Wissenschaften, St. Petersburg. 1087 pp.

Servadei A., 1952 - Hemiptera Sardiniae (Heteroptera et Homoptera Auchenorrhyncha). Redia, 37: 443-478.

SERVADEI A., 1967 - Rhynchota - Heteroptera, Homoptera Auchenorrhyncha. Catalogo topografico e sinonimico, Fauna d'ltalia IX. Ed.Calderini, Bologna. $\mathrm{x}+851 \mathrm{pp}$.

Singer K., Mancini C., 1938 - Contributi alla conoscenza della fauna entomologica della Sardegna. Hemiptera Heteroptera. Memorie della Società Entomologica italiana, 17: 15-20.

SLATER J.A., 1964 - A catalogue of the Lygaeidae of the world. University of Connecticut, Storrs. 1668 pp.

Stichel W., 1938 - Illustrierte Bestimmungstabellen der deutschen Wanzen. (Hemiptera-Heteroptera). Ed.Verlag. Leipzig $439 \mathrm{pp}$.

STichel W., 1959 - Illustrierte Bestimmungstabellen der Wanzen. II. Europa (Hemiptera-Heteroptera Europae), vol. IV. Ed.Verlag. $838 \mathrm{pp}$.

TAmanini L., 1964 - Eterotteri dell'Isola di Pantelleria (Heteroptera). Atti della Società italiana di Scienze naturali e del Museo Civico di Storia naturale di Milano, 103: 65-71.

TAMANINI L., 1973 - Studio sistematico e corologico degli emitteri eterotteri delle Isole Egadi, Eolie e di Ustica. Bollettino della Sedute dell'Accademia Gioenia di Scienze Naturale in Catania, ser. 4, 11(9-10): 9-25.

TAMANini L., 1981 - Gli eterotteri della Basilicata e della Calabria (Italia meridionale). (Hemiptera Heteroptera. Memorie del Museo Civico di Storia Naturale di Verona, 2. Serie. Sezione Scienze della Vita (a: Biologica), 3: 1-164.

WAGNER E., 1958 - Nachträge zur Hemipterenfauna Siziliens (Heteroptera). Bollettino della Società Entomologica Italiana, 88: 139-142.

WALKer F., 1872 - Catalogue of the specimens of Hemiptera Heteroptera in the collection of the British Museum, 8 pt. Printed for the Trustees of the British Museum, London. $202 \mathrm{pp}$.

Nicola Olivieri

via Maestri del lavoro 40, 64100 Teramo, Italia E-mail: nicolao@interfree.it

\section{4 - Acanthocinus griseus (Fabricius, 1792) (Coleoptera Cerambycidae Lamiinae)}

PorTA A., 1934. Fauna Coleopterorum Italica. Vol IV - Heteromera -Phytophaga. Stabilimento Tipografico Piacentino, Piacenza. pp. 220-221.

Prima segnalazione per la Sardegna di una specie già nota per l'Italia di Val d'Aosta, Piemonte, Lombardia, TrentinoAlto Adige, Liguria, Emilia-Romagna, Toscana, Umbria, Marche, Lazio, Abruzzo, Campania, Puglia, Calabria (Sama \& Rapuzzi, 2011).

RePERTI. Sardegna, Lotzorai (prov. di Nuoro), pineta, m 7 s.1.m., 1 ô, 11.VI.2018, F. Musio, C. Sciola leg., A.B. Biscaccianti, F. Fois det. (coll. AGRIS, Azienda San Michele, Ussana). L'esemplare è stato catturato mediante trappola di tipo cross-vane attivata con attrattivo commerciale per coleotteri xilofagi (Gallo Pro Pinowit, prodotto dalla ditta Witasek PflanzenSchutz $\mathrm{GmbH}$ ) ed etanolo al 70\%, nel corso di un monitoraggio di insetti xilofagi alieni.

Osservazioni. Specie a corotipo Euro-Asiatico, diffusa in gran parte dell'Europa, Asia Minore, Caucaso, Asia centrale e settentrionale fino al Giappone. In Italia A. griseus è relativamente localizzato ma in espansione negli ultimi decenni nelle pinete artificiali, verosimilmente favorito dagli incendi (Biscaccianti, 2004 e dati inediti). Si può reperire dal livello del mare alla montagna. La larva si sviluppa su Pinus spp. e secondariamente su Picea spp. (Sama, 1988).

\section{BIBLIOGRAFIA}

BisCACCIANTI A.B., 2004 - Note su alcuni longicorni dell'Appennino umbro-marchigiano (Italia centrale) (Coleoptera, Cerambycidae). Bollettino dell'Associazione romana di entomologia, 59 (1-4): 43-88.

SAma G., 1988 - Fauna d'Italia XXVI. Coleoptera Cerambycidae. Catalogo topografico e sinonimico. Calderini, Bologna, XXXVI + 216 pp. 
SAMA G., RApUZzi P., 2011 - Una nuova checklist dei Cerambycidae d'Italia (Insecta Coleoptera Cerambycidae). Quaderno di Studi e Notizie di Storia Naturale della Romagna, 32: 121-164.

Francesco FOIS, Francesco FODDI Agris Sardegna, Servizio Studi ambientali, difesa delle colture e qualità delle produzioni, Azienda Sperimentale S. Michele, SP 10, Km 2.300, Ussana (CA), Italia. E-mail: frfois@agrisricerca.it

Alessandro B. BISCACCIANTI
Laboratorio di Entomologia ed Ecologia Applicata, Dipartimento PAU, Università “Mediterranea” di Reggio Calabria, Salita
Melissari, Reggio Calabria, Italia.
E-mail: alessandro.biscaccianti@unirc.it

Mauro NANNINI

Agris Sardegna, Servizio Studi ambientali, difesa delle colture e qualità delle produzioni, V.le Trieste 111, Cagliari, Italia. E-mail: mnannini@agrisricerca.it

\section{5 - Monochamus galloprovincialis (Olivier, 1795) (Coleoptera Cerambycidae)}

Wallin H., Schroeder M., Kvamme T., 2013 - A review of the European species of Monochamus Dejean, 1821 (Coleoptera, Cerambycidae) - with a description of the genitalia characters. Norwegian Journal of Entomology 60, 11-38. Prima segnalazione per la Sardegna di una specie già nota per l'Italia di Val d'Aosta, Trentino Alto Adige, Veneto, Friuli Venezia Giulia, Piemonte, Lombardia, Liguria, Emilia Romagna, Toscana, Marche, Umbria, Lazio, Campania, Puglia, Basilicata e Sicilia.

RePerti. Sardegna, prov. Cagliari, Sinnai, pineta, m 152 s.l.m., 1 q, 13.VII.2017, F. Musio \& C. Sciola leg., F. Fois, F. Foddi \& M. Nannini det. (coll. AGRIS, Azienda San Michele, Ussana). Sardegna, prov. Nuoro, Lotzorai, pineta, m -7 s.1.m., 1 9, 3.X.2018, F. Musio \& C. Sciola leg., F. Fois, F. Foddi \& M. Nannini det. (coll. AGRIS, Azienda San Michele, Ussana). Gli esemplari sono stati catturati nel corso di un monitoraggio di insetti xilofagi alieni eseguito mediante trappole di tipo cross-vane e multi-funnel, attivate con feromoni di aggregazione e sostanze ad azione cairomonale.

OSSERVAZIONI. Specie polimorfa ad ampia distribuzione europea e in parte asiatica (dalla penisola Iberica alla penisola Scandinava fino alla Russia Europea e all'Ucraina), rappresenta assieme a M. saltuarius (Gebler, 1830), M. sartor (Fabricius, 1787) e M. sutor (Linnaeus, 1758) una delle quattro specie di Monochamus segnalate per il territorio italiano. Nelle aree di distribuzione $M$. galloprovincialis è presente con due sottospecie: la ssp. pistor nota delle Alpi orientali e la ssp. nominale, rinvenuta nel litorale tirrenico dove ha raggiunto il crinale appenninico, scavalcandolo fino a popolare le pinete artificiali del crinale romagnolo, ricomparendo poi sul Promontorio del Gargano. La sua diffusione è poco chiara per quanto riguarda il versante adriatico, forse per una penetrazione avvenuta da più direttrici; in Venezia Giulia è presente con una forma intermedia tra le due sottospecie (Sama, 1988). Monochamus galloprovincialis riveste importanza fitopatologica non tanto per l'attività trofica che esplica nei confronti dei pini nei quali compie lo sviluppo, ma in quanto potenziale vettore del nematode Bursaphelenchus xylophilus (Steiner \& Buhrer), noto come nematode del pino, specie fitopatogena da quarantena inserita nella lista A2 dell'EPPO, la cui presenza in Europa è già stata rilevata in Portogallo nel 1999 (Sousa et al., 2001) e in Spagna nel 2008 (Robertson et al., 2011; Abelleira et al., 2013).

\section{BIBLIOGRAFIA}

Abelleira A., Picoaga A., Mansilla J.P., Aguin O., 2013 - Detection of Bursaphelenchus xylophilus, causal agent of pine wilt disease on Pinus pinaster in Northwestern Spain. Florida Entomologist, 96(3): 770-780.

ANGELI F., 2012 - Monochamus galloprovincialis (Olivier, 1795) (Coleoptera Cerambycidae) - 537. V.V., A. Segnalazioni faunistiche italiane. Bollettino della Società Entomologica Italiana, 144(1): 44-48. 
Robertson L., Cobacho Arcos S., Escuer M., Santiago Merino R., Esparrago G., Abelleira A., Navas A., 2011 - Incidence of the pinewood nematode Bursaphelenchus xylophlius Steiner \& Buhrer, 1934 (Nickle, 1970) in Spain. Nematology, 13(6): 755-757.

SAMA G., 1988 - Fauna d'Italia XXVI. Coleoptera Cerambycidae. Catalogo topografico e sinonimico. Calderini, Bologna, XXXVI + $216 \mathrm{pp}$

SAma G., Rapuzzi P., 2011 - Una nuova Checklist dei Cerambycidae d'Italia (Insecta Coleoptera Cerambycidae). Quad. Studi Nat. Romagna, 32: 121-164.

Sousa E., Bravo M.A., Pires J., Naves P., Penas A.C., Bonifacio L., Mota M., 2001 - Bursaphelencon xylophilus (Nematoda; Aphelenchoididae) associated with Monochamus galloprovincialis (Coleoptera; Cerambycidae) in Portugal. Nematology, 3, 89-91.

Francesco FOIS, Francesco FODDI

Agris Sardegna, Servizio Studi ambientali, difesa delle colture e qualità delle produzioni, Azienda Sperimentale S. Michele, SP 10, Km 2.300, Ussana (CA), Italia.

Mauro NANNINI

Agris Sardegna, Servizio Studi ambientali, difesa delle colture e qualità delle produzioni,

V.le Trieste 111, Cagliari, Italia.

E-mail: mnannini@agrisricerca.it 